patient. As to migrain, there is no doubt that in all these cases we are dealing with toxemia, and I think that the inves. tigations of Ratchford have shown conclusively that it is not uric acid, but some of the by-products, and I am satisfed that is the underlying condition in most cases of migrain. However earnestly you look for end-organ disease, however you eliminate eye-strain, there will be a very large proportion of headaches in which you will not discover the cause, and which you will fail to relieve.

Dr. Hugh T. Patrick, Chicago-I should like to ask one question of Dr. Gradle. I understood him to say that onesided migrain was due to eye or nose trouble in all cases. If he meant that statement, I wish to very positively and emphatically dissent from it. If he says that one-sided migrain may be due to eye or nose trouble, I agree with him.

Dr. C. C. Hersman, Pittsburg-Quite a number of times in cases of headache thought to be due to eye trouble the head ache was not benefited by correcting the eye trouble. I think, as Dr. Meyer stated, we will find the trouble due to a toxemic condition. In some cases of angioneurotic edema we have excruciating headaches; it is almost impossible to relieve them without very heavy doses of narcotics, and while we have very little light on angioneurotic edema, still I think that is a toxemic condition. In this trouble sometimes the swellings come on close to the monthly period, or we may find it two or three times during the month. A great many of the headaches are attributed to improper food and irregular habits; but if you take these same patients and make them live the most correct life possible for them to live, the attacks will occa. sionally occur. I do not understand the reader as Dr. Patrick understood him. I understood him to say that when we did have a one sided headache it was most likely to come on the side of the bad eye.

Dr. T. D. Crothers, Hartford, Conn.-There are two facts that I think are very prominent. I think all these cases of headache are practically poison and starvation cases. The whole philosophy turns on that, and if you can eliminate these two elements you are successful.

Dr. Charles H. Hughes--The chair would like to remark that the phenomena of headache are too much viewed from one spot rather than from the whole individual. Any one who has had experience with migrain, of which I have had an abundance for twenty years, knows what constitutes migrain. In my own case I was in the habit of forcing mental action to the utmost limit by the use of coffee. For twenty years of my life I used a half-gallon of coffee between 9 o'clock and bed-time, and forced myself to a condition of wakefulness. I always slept in spite of coffee, but I learned afterward that I did not sleep in each twenty-four hours quite so much as was necessary for daily recuperation. My migrain came upon me in this way first at the end of thirty days I was obliged to retire hors de combat for about one day ; could not tolerate the light; so hyperesthetic that I could not tolerate sensation of any kind. The greater part of that day was spent in misery and retirement. Then the conservatism of nature asserted itself; the sleep which followed, and which was more than I was in the habit of getting, was a compensation, and I was restored to a more normal condition. When it became my misfortune to preside over the fortunes of a lunatic asylum these migrain attacks came on every week, and the only thing that conserved my vitality at all was the very fact that it knocked me out so completely that I could not do anything for nearly one day a week. The toxemic theory has found a very satisfactory lodg ment in my mind. I have no headaches now, and have not had for fully ten years; but I have learned to take care of mysel better. The amount of brain strain that a man puts upon himself is sufficient to bring about this condition of the accumulation of toxins, and they cause the trouble, as I think is no doubt the case in our epileptic conditions; but it is necessary to have an organism that is predisposed. Exophoria, esophoria errors of accommodation and refraction of various kinds are associated conditions, and they tend, where you have a neuropathic condition that gives you the headache, to make the latent condition active; but I do not think, as the ophthal mologists assert, that they are every, the sole, exclusive and only cause. As Dr. Brower said, the removal of whatever source of irritation that may exist is good therapy; it is good practice in neurotherapy as in every other department of med icine; but to assume that these migrains are the result of just one thing is a mistake in my judgment. As to medical treatment of my headaches, I learned to use bromid of potassium afterward bromid of sodium, and I learned to get more rest and to adjust the expenditure of nerve force to my capacity for daily accumulation. The same condition that will give you a dia. betic urine will, in the cortex, in the meninges, give rise to a headache; but it seems to me that this whole subject is a sub. ject concerning the treatment of the individual and his habits. Irritation in the eye will bring out a latent headache.

Dr. Harold N. MoYer, Chicago-Is it not true that ninetenths or more of the migraine practically cease with advancing years?

Dr. Hughes-That is a fact, I think. At 45 years of age the majority of people cease to have migrain. At that time of life when they begin to understand what life is.

Dr. MOYER-And when their blood-vessels get a little harder? Dr. Hughes - That may possibly be the explanation.

Dr. GradLE -I was not presumptuous enough to $\operatorname{come}$ before a Section of Neurologists with a paper on the treatment of headache. My paper merely had to do with the characteristics of some headaches which were relieved by local treatment. I wished to present those symptome by which the peripheral origin can be determined, and relief obtained. Dr. Patrick did not understand me correctly. I said in the paper that in every case of one-sided headache the cause was either a one-sided intracranial lesion, or an affection of an organ of special sense on the same side. I base this on my own expe. rience. I have never seen an exception to this rule. I was always able to trace such a condition to some affection of one side of the head, with this exception, however, that those cases of migrain which alternate regularly between the right and the left side do not come in the category; they are not due to one-sided lesion, or any other lesion. I have been misunstood as to the importance of the peripheral lesion in causing headache, especially migrain. I stated in my paper explicitly that in every case of headach $\theta$, not only the alleged peripheral causes but also the condition of the nervous system needed investigation. I added, furthermore, that there are some peripheral causes so potent that they would introduce headache in anybody, no matter whether they be in good health or not. For instance, a severe injury to the eye, causing inflammation, will invariably produce headache; or severe coryza extending into one of the sinuses of the nose will cause headache as a regular consequence, no matter how perfect the nervous system of the individual. On the other hand, an astigmatism of one diopter or thereabout will not necessarily give eye symptoms to a normal individual, whereas if the individual is run down, or other depressing factors are at work, he will have eye symptoms.

The question has been asked about the frequency of astig. matism as the cause of migrain. That the oculist can not answer, as his cases are selected. In the great majority of cases that were sent to me l was able to demonstrate an ocular origin, but, of course, only those cases came to me where a peripheral origin was suspected. I presume that a large number of cases of migrain have no peripheral origin. The proof is very definite that the peripheral irritation is sufficient to bring on these attacks. That other factors are present, also, there can be no reason to doubt, because of the periodicity of the attack. There must be some other factor. My paper did not deal with the pathology of headaches; I did not refer to these factors, and 1 am not at all sure that the toxemic theory is sufficiently proven.

In answer to Dr. Hughes' query as to cases of astigmatism coming on in school children while they are at work, and dis. appearing when they leave echool for the term, I would say that the astigmatism does not really disappear; the astigmatism is stable in the majority of its conditions; the others are very rare cases. The strain is lessened when the child is at liberty. I consider it very one-sided and altogether wrong to finish the work by prescribing glasses, and not to consider the other factors at all. Of course, if the glasses relieve there is no reason why they should not be given.

\section{EYE SYMPTOMS OF BRAIN TUMOR.}

Presented to the Section on Neurology and Medical Jurisprudence at the Forty-ninth Annual Meeting of the American Medical Association, held at Denver, Colo., June 7-10,1898.

BY EDWARD:JACKSON, A.M., M.D.

$$
\text { OF DENVER, COLO. }
$$

EMERITUS PROFESSOR OF DISEASES OF THE EYE IN THE PHILADELPHIA POLYCLINIC, FORMERLY SURGEON TO WILLS' FYE HOSPITAL. PHILADELPHIA.

\section{OPTIC NEURITIS.}

Among the eye symptoms of brain tumor we naturally think first of optic neuritis, the most striking and significant symptom in cases of this class. Probably not a few practitioners have associated optic neuritis chiefly or wholly with intracranial disease or 
intracranial growths. But while it is right that great importance should be attached to this symptom, occurring as it does in 80 or 90 per cent. of all cases, it is necessary to point out that its discovery is far from establishing a diagnosis of brain tumor. It is of very little value to indicate the location of the tumor, and it is worthless as regards revealing the nature of the growth. But it does show with great probability that brain symptoms accompanying it are due to serious organic disease. Even here, however, its indication is not absolute, for, in common with these symptoms, it may arise from different kinds of poisoning.

It has been a matter of some surprise in going over a series of my cases of marked optic neuritis, to find how exceptional has been its connection with intracranial growths, and how many cases occur in connection with other diseases. Thus, among 27 cases, but 17 were probably connected with serious organic diseases of the brain and its membranes, and only 8 of those were-probably five or certainly three-cases of brain tumor. The others arose in connection with renal and vascular diseases, syphilis, various toxic agents, chiefly lead and alcohol, disease in the orbit, or of the ethmoidal and sphenoidal sinuses, or were of such obscure etiology as to be classed as idiopathic.

Unfortunately, optic neuritis due to brain tumor presents no especial characteristics that separate it sharply from optic neuritis due to these other causes. Indeed, it is sometimes difficult to distinguish it from such an entirely different condition as the optic neu. ritis due to eye-strain, or from the protruding opaque optic disc that occurs in rare cases, probably as a congenital anomaly. So far is optic neuritis from fixing the underlying pathologic condition as one of brain tumor, that in determining the cause of the optic neuritis the other symptoms of the case have to be carefully considered. Still the ophthalmoscopic appearances do give certain indications of the cause of neuritis, which are of value in reaching a diagnosis.

Let us contrast the neuritis due to the various other conditions mentioned with the typic optic neuritis of brain tumor. Such typic neuritis is characterized by great swelling, sometimes 10 or $12 \mathrm{D}$ (3 m.m.), abruptly limited, at no great distance from the margin of the disc, with arteries narrowed, veins dilated and very tortuous, and small vessels much enlarged, but not very numerous, because scattered through the swollen tissue; and small, flame-shaped hemorrhages upon or near the papilla. The other portions of the fundus, except for the alteration in the vessels, are normal, or present changes which are slight as compared with those at the disc, although to this last statement there are very important exceptions.

In contrast with the above: Swelling and discoloration of the disc, occurring as an anomaly, are never of very high degree. No such recorded case showed more than $4 \mathrm{D}$. swelling of the optic papilla, and in none that I have encountered was the most and prominent portion of the swelling more than $3 \mathrm{D}$. in front of the level of the neighboring fundus. Again, even with the higher degrees of such swelling the retinal vessels are normal; and the other parts of the fundus are normal, or exhibit no lesions like those of neuro. retinitis.

Optic neuritis, duc to eye-strain, is more common, but in this also the swelling is limited. The disc may be very red and yet the most prominent vessels be not over $1 \mathrm{D}$. in advance of the fundus. In this form, too. the larger vessels are but slightly altered, and the fine vessels of the optic disc are very much more numerous, but are not particularly irregular in caliber or very tortuous.

Under idiopathic optic neuritis, or optic neuritis of unknown causation, I should prefer to place the unusual but not very rare cases that have been ascribed to cold, rheumatism, disturbance of menstruation, etc. The ophthalmoscopic appearances resemble those of optic neuritis due to eye-strain, except that the swelling may be greater and the retinal circulation more interfered with. Optic neuritis of this kind is usually acute, and tends to early and comparatively complete recovery. The field of vision does not present great concentric or irregular contraction as compared with the ophthalmoscopic appearances, although the blind spot may be markedly enlarged and irregular. I have met with one case in which such optic neuritis, with great impairment of vision recurred, yet was followed by almost complete restoration to normal.

Optic neuritis in connection with gummatous growths is rather frequent in the tertiary stage; but it may also occur as one of the earlier secondary lesions of syphilis. I have seen it within three months after the initial lesion. In this stage it presents the clinical appearances of idiopathic optic neuritis and is rather promptly amenable to treatment. Of the form of retro-bulbar optic neuritis that leads to central scotoma without marked ophthalmoscopic changes it is unnecessary here to speak. But in rare cases, certain toxic agents, especially lead, may cause a form of optic neuritis that closely simulates that due to brain disease. It is usually chronic, and attended with great swelling and corresponding disturbance of circulation, while the general condition of the fundus remains, as in the optic neuritis of brain disease, comparatively good. I know of no signs by which one may, with the ophthalmoscope, discriminate between this form and the optic neuritis due to brain tumor. The diagnosis between must be based on other symptoms, that should be constantly borne in mind in connection with this subject.

But most frequently the doubt as to the significance of optic neuritis rests upon the resemblance of the lesions in certain cases of renal vascular disease, to those connected with brain tumor. The forms of optic neuritis due to these two causes may in typic cases be quite distinct, and in the majority of cases there is exhibited a certain divergence of type. But in some it is absolutely impossible to decide from the ophthalmoscopic examination to which class the particular case belongs; and in some, neuritis due to the one cause inclines rather to the clinical type of the other. The typic differences are these: Optic neuritis due to renal and vascular disease is more apt to be attended by bemorrhages and patches of exudation throughoui the fundus; is more apt to be accompanied by the stellate grouping of white spots at the macula; is more apt to be associated with marked irregularities in the caliber and appearances of the vessels, apart from dilatation and tortuosity of the veins. Still, optic neuritis from brain tumor may be accompanied by general retinal hemorrhages and considerable areas of retinal exudation, especially at the macula, or between it and the disc (Harlan). Even the stellate group of white spots in the macula formerly regarded as pathognomonic of albuminuric retinis may be included among the retinal lesions connected with brain tumor (de Schweinitz). 
With very extensive retinal lesions one may say that in all probability the trouble is of renal-vascular origin. With a complete absence of them, one may say that violent optic neuritis is probably due to organic disease within the cranium, but the possibil. ity of confusion should not be lost sight of. Bearing it in mind, examination of the urine will usually show to which class the case belongs.

Given then a case of optic neuritis that can be excluded from the class we have just considered, its existence indicates with great probability the presence of grave organic disease within the cranium. Further than this we can scarcely go. It will be impossible to say from examination of the eyes that the cause is a brain tumor, a meningitis, or a cerebral abscess. The rapidity with which the neuritis runs its course will, however, in some cases give a valuable indication. Although Gowers shows that chronic neuritis never results from a rapidly growing tumor, in a tumor of very slow development the neuritis may be rather more chronic than from any other cause, slowly increasing for many months before vision is seriously impaired and running a course of years before it ends in atrophy.

\section{OPTIC ATROPHY.}

This is usually consecutive, coming on as the termination of optic neuritis. As such it has the same significance, and requires the same caution in exclud. ing other possible causes.

Among 40, that I regarded as cases of consecutive atrophy, 13 were apparently due to organic intracranial disease; of these 3 were probably cases of tumor. The tendency in all cases is for optic neuritis to pass into atrophy, but in connection with brain tumor neuritis runs such a chronic course, that a large proportion of patients die before the atrophy predominates. The rapidity of the change to atrophy is usually related to the rapidity of the development of the neuritis. Marked permanent impairment of central vision and contraction of the field of vision are associated rather with the atrophy than with the neuritis. For a considerable time after atrophy is well. marked the evidences of previous neuritis remain unmistakable and not easy to overlook. But if the case be very chronic it is possible that the appearances which characterize the atrophy as consecutive, may be so trifling as to readily escape notice. These appearances are at first the remaining symptoms of neuritis; especially the swelling, now growing pale, the opacity which hides the disc outline, and the patches of exudate in the retina. Later the head of the optic nerve becomes clearly outlined, although still so opaque that the vessels can not be traced far into it. The vessels diminish in size, the veins as well as the arteries, though the former remain relatively large as compared with the latter; the vessel walls are more noticeable than normal, the vessel being sometimes accompanied by white streaks well out into the periphery. Patches of former retinal exudate may now appear as white degeneration, or only as areas of disturbed retinal pigment.

Around the disc may almost always be noticed some disturbance and absorption of the choroid, which is apt to be greatest at the upper and lower margins of the disc. This location distinguishes it from the somewhat similar choroidal changes due to previous eye-strain, which are generally most pronounced at the temporal margin of the disc. In rare cases sim. ple atrophy, that is, atrophy without previous neuritis, has occurred in the course of brain tumor. It is due to pressure upon the optic nerves, chiasm, or tracts, or to direct destruction of the optic radiations or centers. Having this origin it becomes of considerable localizing value.

CHANGES IN THE PUPIL.

With impairment of accommodation changes in the pupil may occur as effects of direct involvement of the nerve tracts concerned. Sudden temporary disturbances may occur here, as they occasionally do in other forms of chronic organic disease of the brain and cord; but they have no especial significance with regard to brain tumors. The Wernicke symptom or reaction, or want of reaction, of the pupil to light thrown upon the blind half of the retina has in a few cases great localizing value. It throws no light upon the nature of the lesion which produces it. In attempting to elicit this symptom, especially when the vision is greatly impaired and the field contracted in all directions, it is sometimes necessary to throw the light into the eye from a point not greatly removed from the visual axis. In this way contraction of the pupil to light may be obtained, when, if the light were simply let fall toward the periphery of the retina, the result would be entirely negative. Of course, the nearer the light is brought to the direction of the visual axis, the greater the care that must be observed to insure that the pupillary reaction is not brought about by light falling upon the macula. Where the field of vision is still wide and the pupil reacts strongly to light, care should be taken that the light does not reach the seoing half of the retina through the sclera covering it, in sufficient quantity to provoke the contraction of the pupil, when, from light falling on the blind half of the retina, no contraction would occur.

To guard against this source of error I commonly use the light as reflected from the ophthalmoscope mirror, which can be concentrated in a small area upon the face and then turned so as to fall upon only the front and blind side of the eye. For instance, suppose a case of right homonymous hemianopsiathe left half of each retina blind-the source of light being behind the patient, the ophthalmoscope is held to the patient's right, as compared with his visual axis. The light is properly concentrated upon the brow or the nose. and then the mirror turned so that the light area will fall upon the pupil and portion of the sclera to the left of it, the sclera to the right of the cornea remaining in darkness.

PARESES OF THE EXTRAOCULAR MUSCLES.

In connection with brain tumor these are chiefly of value in the matter of localization, and the exact sig. nificance of each of the forms of extraocular paralysis in this direction need not now be rehearsed. In a general way such lesions, due to brain tumor, are more slow in their onset, and are longer in reaching complete development than those due to other causes, even perhaps those due to nuclear degeneration. But not much importance should be attached to this, since in connection with brain tumor their development may be entirely sudden.

A subject that has not attracted the attention it should have, and which will probably profitably repay careful study, is that of paresis or transitory disturb. ances of the extraocular muscles, which occur as dis. tant symptoms with brain tumor, as they do with otler 
forms of organic disease, and which may be one way that the new growth causes such symptoms as head. ache and dizziness. In some cases at least they arise early, having probably much the same origin and significance as the mental disturbance, and the tendency to forced positions of the limbs, or involuntary movements that are sometimes seen. They are at least worth studying with the Maddox rod, the tendencies to deviation of the visual axes being taken when the eyes are turned toward various parts of the visual field. NYSTAGMUS.

Although rarely accompanying blindness from cataract or corneal opacity, nystagmus is encountered in a large proportion of all cases of blindness from disease of the central nervous system. It is generally present when complete blindness has existed for many months in the course of brain tumor; but its occurrence in connection with other intracranial lesions robs it of especial diagnostic importance. It is probably more common and appears earlier in tumor of the cerebellum. In a few cases it appears while there is still fair sight, generally in connection with other disorders of the extraocular muscles.

IMPAIRMENT OF VISION.

Impairment of central vision and limitation of the field of vision may be of importance in calling attention to the existence of serious organic disease. The exactness with which they can be estimated quantitatively, gives them value as indieating the progress of the case. In the large majority of cases, however, they depend upon the optic nerve lesion and indicate its progress rather than that of the original disease. In a few cases where the affection of sight is due to direct involvement of the visual tract the changes in the field of vision become of the highest significance as a localizing symptom. Transient impairments may occur as a distant symptom, having the same significance as the mental disturbances and hysteroid symptoms.

Impairment of color perception can not be regarded as of a special localizing value. As Holden has shown, it may be merely a more delicate indication of the injury to the visual conducting apparatus, before this injury has become sufficient to interfere with the perception of form.

THE STIGMATA OF NERVOUS SYPHILIS.

Presented to the Section on Neurology and Medical Jurisprudence, at the Forty-ninth Annual Meeting of the American Medical

Association, held at Denver, Colo., June 7-10, 1898.

BY WILLIAM J. ROTHWELL, M.D.

PROFESSOR OF NEUROLOGX, GROSS MEDICAL COLLEGE. DENVER, COLO.

Specific lesions of the nervous system produce localizing symptoms similar in character to those which result from non-specific lesions having the same anatomic seat. Partial epilepsy of gummatous origin or hemiplegia from syphilitic thrombosis of the central arteries has no distinguishing features, per se: associate symptoms alone reveal its real nature. To describe these characteristic symptoms and to emphasize their importance in the diagnosis of cerebral syphilis in particular, is the object of my paper. A brief reference to the salient pathologic features of specific nervous disease is essential for the better understanding of the subject.

It is well known that syphilitic inflammation affects primarily the blood-vessels and meninges of the neu- raxis. While no part of this extensive territory is exempt from specific invasion, the most vulnerable parts are the convexity of the hemispheres, especially in their anterior portion, and more frequently still the base of the brain from the chiasm to the annular protuberance, involving the apparent origin of the cranial nerves. In both the meninges and the blood-vessels these gummatous products occur in two different states of aggregation, the on $\theta$ focal, the other diffuse. In the pia-arachnoid they are generally circumscribed; in the dura, on the contrary, diffuse gummatous infil tration is the rule. In the arterial walls they are also found as plaques or patches resembling atheroma, or as extensive infiltrations of the tunica intima. $R_{\theta}$. markable peculiarities of this tissue are its rapid growth, low vitality and the rapidity with which it undergoes retrogressive changes.

Since the meninges envelop every part of the encephalo-spinal axis, and the blood vessels traverse all its conducting paths and functional centers, a great number and variety of symptoms are to be expected in a general infection like syphilis, with a special pre. dilection for these tissues. The symptoms of specific involvement of the nerve centers are, therefore, apt to be not only multiple but multiform. The initial manifestation may be, indeed, single, but if the disease be left to pursue its own course, evidence of multiple lesions will soon appear. Thus a double hemiplegia with aphasia, depending as it must upon two foci of disease, points to a specific origin, while a right hemiplegia, with or without aphasia, being of necessity due to a single lesion, indicates a common source. Of like significance is the association of symptoms of involvement of the third cranial nerve on one side with hemiplegia of the opposite side. A very striking exception to the law of multiplicity of lesion is the isolated loss of the iritic reflex to light, which is considered by Gowers to be an important indication of cere. bral syphilis.

We have already called attention to the fact that the morbid product of syphilitic inflammation, the so-called gummatous exudate, grows rapidly and as rapidly disappears. The symptoms depending upon such lesions must possess the same transitory character. And this is true of all disturbances resulting from the specific process itself. Paresis, aphasia, strabismus and hemianopsia (when not associated with hemiplegia) of acute onset and brief duration, point almost unerringly to the primary effects of gummatous exudations. We must recollect, however, that these products may damage the nerve structures by pressure or by interference with their blood-supply, and that these secondary lesions belong in the class of necroses or degenerations, of which the effects may not be transitory but permanent.

Syphilitic arteritis is a curable malady, but when arterial thrombosis occurs with consequent ischemia and necrosis of that vascular area, the pathologic process is no longer specific, and the clinical history merges into that of common lesions, the disability becomes more or less permanent. Gummatous exudates, compared with other new formations, develop rapidly. and this pathologic feature gives to many specific lesions a characteristic onset. Cerebral hemiplegia of common origin has, as a rule, a sudden onset. Gow. ers claims that a sudden onset is characteristic of all vascular paralysis. When, however, the hemiplegia depends upon syphilitic thrombosis the onset is rather subacute than sudden. It is preceded by headache in 